BP-PP-1-2

\title{
Biologic behavior of resected BRCA-mutated pancreatic cancer: Comparison with sporadic pancreatic cancer and other BRCA-related cancers
}

\author{
Sung Hyun KIM, Chang Moo KANG*
}

Department of Hepatobiliary and Pancreatic Surgery, Yonsei University College of Medicine, Seoul, Korea

Introduction: Since margin-negative resection is essential for the cure of pancreatic cancer (PC), early detection of PC is important. Although PC is the third most common cancer associated with BRCA1/2 mutations, clinical research regarding BRCA mutations in resected PC are rare. In this study, we investigated the oncologic characteristics of resected PC with BRCA mutation to suggest management strategies.

Methods: We retrospectively reviewed data from 493 patients who were confirmed to be pathogenic BRCA1/2 mutation carriers between January 2007 and December 2019. We investigated the oncologic characteristics of PC patients by comparing them with resected sporadic PC and other BRCA-related cancer groups (breast cancer, ovarian cancer, and others).

Results: Ten BRCA mutation carriers (2.0\%) experienced PC, and PC onset was significantly later than that of BRCA-related breast cancer (age: breast vs. pancreas, 45.0 vs. 53.5 years; $p=0.050$ ). Six patients underwent pancreatectomy and their long-term survival outcomes did not differ from those of sporadic PC patients (disease free survival: BRCA1/2 vs. sporadic, 10.0 months vs. 9.0 months; $p$ $=0.504$; overall survival: BRCA1/2 vs. sporadic, 29.0 months vs. 35.0 months; $p=0.520$ ).

Conclusions: BRCA-mutated PC occurs later than BRCA-mutated breast cancer. Active genetic testing to identify BRCA1/2 mutation carriers at the onset of breast cancer and continuous long-term surveillance of these patients can provide opportunities to detect BRCA-mutated PC at a resectable stage. 